\title{
Optimisation of bronchial brachytherapy catheter placement with a modified
} airway stent

\author{
M.P. Kennedy*, C.A. Jimenez*, J. Chang" , F. Fossella", G.A. Eapen* and R.C. Morice*
}

ABSTRACT: Brachytherapy is a modality of treatment available for lung cancer with tracheal involvement. Correct positioning of the brachytherapy catheter is vital to the optimisation of treatment effect and reduction of complications. Normal airway anatomy and tumour location can make this positioning difficult.

The current study presents the case of a 65-yr-old male with invasive tracheal squamous cell carcinoma of the anterior main carina involving the proximal left and right bronchus.

The patient was successfully treated with brachytherapy using a novel modified airway stent with a traversing suture for positioning and stabilising the brachytherapy catheters and maximisation of the radiation effect.

This simple yet innovative modification of readily available bronchoscopic equipment permits approximation and fixation of a brachytherapy catheter to any part of the proximal airway. Further analysis of this technique, including a prospective controlled trial, is planned.

\section{KEYWORDS: Brachytherapy, bronchoscopy, lung cancer, radiotherapy}

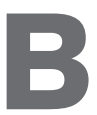

ronchoscopic brachytherapy is an outpatient treatment effective for endoluminal and paraluminal lesions; however, optimal positioning of brachytherapy catheters may be difficult $[1,2]$. This case study describes the use of a novel modified airway stent for positioning and stabilising brachytherapy catheters in a patient with an anterior carinal lesion. It is hoped that detailing this technique helps in the management of other patients with similar cancer presentations.

\section{CASE REPORT}

A 65-yr-old male had originally presented to an external institution with hiccupping 14 months previously. Investigations identified a $4-\mathrm{cm}$ right upper lobe squamous cell carcinoma abutting the mediastinum, a separate squamous cell carcinoma in situ at the main carina and negative mediastinoscopy for nodal involvement. Using tumour, node, metastasis (TNM) staging, the patient was staged as having a T4NOM0 right upper lobe lesion with a synchronous carinal lesion and treated with definitive chemotherapy and radiation (total dose $60 \mathrm{~Gy}$, with 55-60 Gy delivered to the carina). His right upper lobe lesion gave an excellent radiographic response; however, the carinal lesion appeared to progress on bronchoscopy. He was therefore referred to the University of Texas MD Anderson Cancer Centre (Houston, TX, USA).

Bronchoscopic evaluation identified thickening of the main carina (fig. 1a); however, on autofluorescence bronchoscopy, a grossly abnormal class III lesion (red/green ratio of 1.49) was noted draping over the anterior aspect of the main carina (fig. 1b). The lesion extended 5 and $3 \mathrm{~mm}$ down the left and right main bronchus, respectively. Biopsy confirmed residual squamous cell carcinoma. An Olympus $20-\mathrm{MHz}$ radial probe ultrasound catheter (Olympus, Tokyo, Japan) identified cartilage disruption at the carinal lesion. No lymphadenopathy was identified using an Olympus UC160F ultrasonic video bronchoscope (Olympus). The patient was therefore restaged as having locally invasive inoperable carinal squamous cell carcinoma.

Based on the ultrasound evidence of tracheal wall invasion, it was felt that the patient would not be an optimal candidate for either endobronchial laser or photodynamic therapy. Considering the
AFFILIATIONS

Depts of *Pulmonary Medicine,

\#Radiation Oncology, and

"Thoracic Oncology, University of Texas MD Anderson Cancer Centre, Houston, TX, USA.

\section{CORRESPONDENCE}

C.A. Jimenez

Dept of Pulmonary Medicine University of Texas MD Anderson Cancer Centre

Unit 403

1515 Holcombe Boulevard

Houston

TX 77030

USA

Fax: 17137944922

E-mail: cajimenez@mdanderson.org

Received:

July 032007

Accepted after revision:

August 232007

STATEMENT OF INTEREST

None declared.

European Respiratory Journal

Print ISSN 0903-1936

Online ISSN 1399-3003 

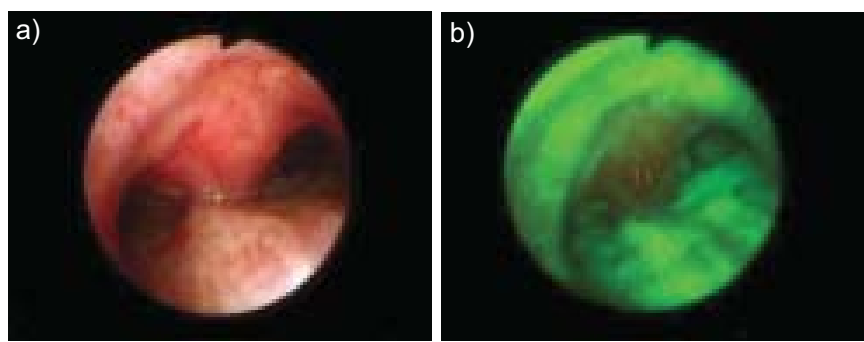

FIGURE 1. Bronchoscopic evaluation of a 65-yr-old male with nonsmall cell lung cancer: a) light; and b) corresponding autofluoresence image. These images display a carinal lesion with increased red/green ratio.

patient had received 55-60 Gy external beam irradiation to the carina, it was crucial that the potential toxicity of additional radiotherapy be minimised while delivering an adequate dose of radiation to the target. A decision was made to proceed with brachytherapy. Two brachytherapy catheters were used, one in each bronchus (fig. 2), due to the involvement of both main bronchi. However, the catheters tended to drift with gravity and may have caused overdose to the uninvolved posterior tracheal wall. A modified silicone stent (cut to $2 \mathrm{~cm}$ ) with a suture traversing its upper aspect was placed in the distal trachea using rigid bronchoscopy. The brachytherapy catheters were then threaded through this loop during placement, resulting in improved catheter positioning, with close approximation to the anterior airway wall in the right and left main bronchus (fig. 2). The catheter position was confirmed using fluoroscopy and flexible bronchoscopy. The treatment target included the $1-\mathrm{cm}$ anterior carinal lesion and $5 \mathrm{~mm}$ of extended disease in both sides of the main bronchus. An additional $1-\mathrm{cm}$ margin was given both distally and proximally. Intraluminal brachytherapy (15 Gy) using a caesium source was delivered to a depth of $7.5 \mathrm{~mm}$. The patient received additional intraluminal brachytherapy to another 10 Gy using the same technique 1 month later. Follow-up with bronchoscopy 2 months later identified an improved appearance of the carinal lesion, and multiple biopsy specimens were negative for cancer.

\section{DISCUSSION}

The inverse square law governs the physical characteristics of the radioactive isotopes used in brachytherapy, i.e. the dose rate decreases as a function of the inverse square of the distance to the source centre. Therefore, it is desirable that the catheter optimally approximates the target lesion. With the normal human trachea measuring $2-2.5 \mathrm{~cm}$ in diameter, catheters can drift away from the target lesion and lead to both a decrease in treatment efficacy and an increase in normal

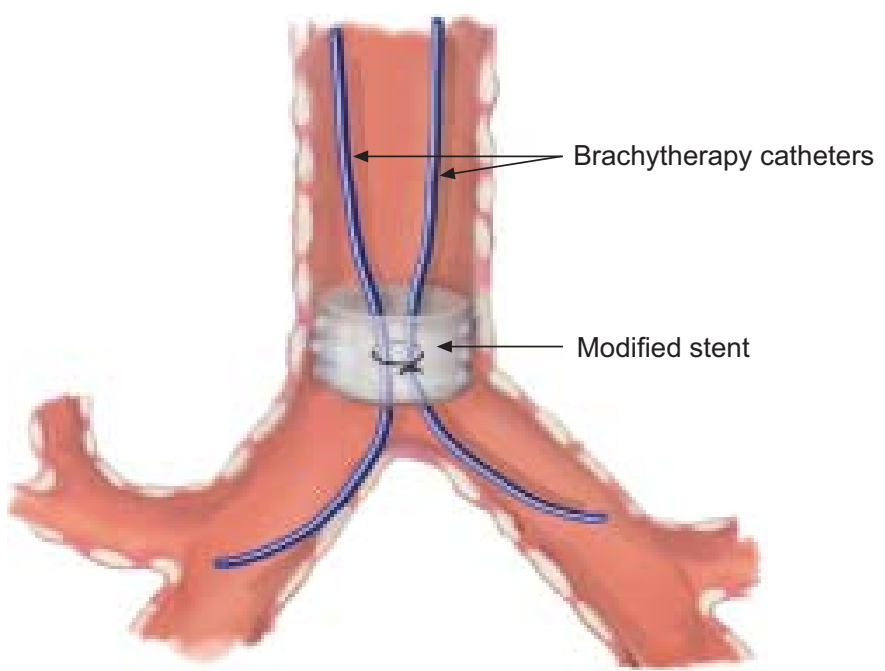

FIGURE 2. The modified airway stent with suture allows anterior fixation of catheters adjacent to the target lesion.

tissue exposure, with attendant complications, such as radiation bronchitis. In this patient, factors such as the normal airway calibre and anterior location of the lesion made accurate positioning of the catheters more challenging.

Centring devices, such as sheaths, cages and balloons, have been used to improve catheter position [3]. Modified applicators have also been used; however, they are not widely available [4]. Use of a modified silicone stent is a simple yet innovative procedure that allows approximation and fixation of the brachytherapy catheter to any part of the proximal airway. The equipment required is widely available and no additional training is required. Further analysis of this technique, including a prospective controlled trial, is planned.

\section{REFERENCES}

1 Raben A, Mychalczak B. Brachytherapy for non-small cell lung cancer and selected neoplasms of the chest. Chest 1997; 112: Suppl. 4, 276S-286S.

2 Vergnon JM, Huber RM, Moghissi K. Place of cryotherapy, brachytherapy and photodynamic therapy in therapeutic bronchoscopy of lung cancers. Eur Respir J 2006; 28: 200-218.

3 Nag S, Kelly JF, Horton JL, et al. Brachytherapy for carcinoma of the lung. Oncology (Williston Park) 2001; 5: 371-381.

4 Nomoto Y, Shouji K, Toyota S, et al. High dose rate endobronchial brachytherapy using a new applicator. Radiother Oncol 1997; 45: 33-37. 